\title{
A cross Layer O ptimized Reliable Multicast Routing Protocol in Wireless Mesh NETWORKS
}

\author{
Mijahed Nasser Aljober, R.C.Thool \\ Department of Information Technology, SGGS IE \& T, Swami Ramanand Teerth \\ Marathwada University, Nanded, Maharashtra, India
}

\begin{abstract}
The Optimal way to create a protocol in Wireless Mesh Networks is to take into account a cross layer due to the interference among wireless transmissions. In this paper, we focus on designing and implementing a reliable multicast protocol called Mesh Reliable Multicast Protocol (MRMP). A recovery tree built dynamically which is joining with the multicast routing tree. Using the recovery tree the packet losses are repaired locally. This Cross layer Technique between network layer (multicast routing) and transport layer (reliability) using simulation results prove the effectiveness and optimization of cross layer in WMNs compare with the conventional layer.
\end{abstract}

\section{KEYWORDS}

Multicat routing, reliable, WMN, Optimization, Cross layer Optimization.

\section{INTRODUCTION}

The main requirement for many applications, such as stock quotes, electronic newspapers, distribution of software, billing records, and medical images is guarantee reliable delivery.

A WMN more concerned with scalable end-to-end throughput and satisfactory quality of service (QoS) to deliver heterogeneous traffic which is differ from wireless sensor network and ad hoc network. Thus, better to optimize the network performance of WMNs across layers protocol [1]. In the traditional network based on a layering approach on the protocol stack. In this modular approach , fundamentals functionalities such as routing (network layer), access to the medium (link layer), congestion control (transport layer) are performed separately, This has proven to be a very powerful and successful paradigm for wired networks, since the infrastructure is based on links with constant capacity and high degree of reliability.

A cross-layer design approach on the other hand, is one that utilizes function across different layers of the protocol stack. Recently several studies prove that cross-layer designs can yield significant performance gains. The penalty that has to be paid for increasing system performance by deploying cross layer designs is the complexity and communication overhead[1].

In this paper we describe a cross layer reliable protocol with assistance of MeshSPT multicast routing protocol[2], this protocol solve the implosion and exposure problems and repair loss packet by recovery tree. The remaining of this paper is as following: in section 2 we present 
International Journal of Distributed and Parallel Systems (IJDPS) Vol.4, No.6, November 2013

related work, in section 3 we explain in details the MRMP protocol, experimental results will be explained in section 4 and conclusion will be explained in section 5 .

\section{RELATED WORK}

Yuan et al. [3] propose a cross-layer optimization framework that scales the demand of network flows at the network layer and the supply of link capacities at the physical layer in order to find high throughput paths. Furthermore, Rong et al. [4] have proposed a Traffic Engineering and preferred admission control policy for QoS Multicast routing in WMNs. However, They considered only mesh backbone. Whereas backbone mesh routers and end-user mesh hosts have to be considered and needed to discussed in QoS multicast mechanism. Ehsan Pourfakhar et al. [5] in case of route disconnection or node use artificial neural network to predict that. To avoid fault occurrence This prediction allow to recover the network. They also present an efficient optimized multicast protocol based on a reliable effective framework to solve the problem of load balancing and to enhance the QoS in multicast communication among Internet hosts and Mesh hosts in WMNs dynamically.

\section{THE PROPOSED PROTOCOL (MRMP)}

\subsection{BRIEF}

In MRMP protocol we use tree-based hierarchical approach to solve the problem of ACK implosion, grouping the receivers based on multicast sub-trees which is rooted at the mesh routers, which called Specified Routers (SRs). Any nesh router in the multicast session and has at least two branches will be chosen as SRs. Each SR will polish the data packets, also collect the status message from the receiver in the same sub-tree, then retransmit repair packet to the receivers (if it has) and report the status to upper level. MRMP protocol also solve the problem of losing packets in multicast session.

A cross-layer design is used in MRMP protocol, which the data packets can examine by routers to get the sequence numbers.

\section{The protocol steps:}

1. Using the multicast tree Sender $\mathrm{S}$ multicasts data packets to all receivers.

2. If there is later retransmission on the time of receiving the data packets, SRs will retransmit the packets again.

3. At regular intervals status messages (contain some information about the packets which have been received successfully) sent by each receiver to its corresponding SR.

4. Based on status message, The packets retransmitted which determine by SR. Locally in the sub-tree the repair packets multicasts. The SR sends its status messages to the upper level SR. same procedure executes again by SR upper level.

5. Multicasts new packets by sender $\mathrm{S}$.

\section{EXPERIMENTAL RESULTS}

In this section, we explain the performance of network in term of throughput, average End-to-End delay and packet delivery ration by simulation results to prove the achievement of cross layer optimization compare with traditional layer in WMNs . 
International Journal of Distributed and Parallel Systems (IJDPS) Vol.4, No.6, November 2013

\subsection{Performance Metrics}

Following the evaluation metrics:

- Packet delivery ratio (PSR) : define as the number of received packets to the number of packets transmitted.

- Average throughput: define as the sum of the throughput of all flows.

- Average end-to-end delay(EED): define as the average delay of packets received from end to another.

\subsection{Simulation SETTING}

The performance of proposed protocol is carried out using Glomosim [6], the same parameters and conditions of [2] were applied. The underlying multicast routing protocol is the MeshSPT protocol. We added some functions to MeshSPT so that it can handle ACK and RTT MEASURE packets and retransmissions of repair packets. We measure the PDR, the average throughput, and the average EED as the number of receivers in a multicast group varies. Each experiment was run five times using different random seed numbers, and collected statistics were averaged over those runs.

\subsection{RESULTS AND ANALYSIS}

Experimental results will be shown in the Figure 1,2. Based on the result we have the following observations:

1. Rreceivers have a PDR of $100 \%$, in both experiments. Means all the data packets are delivered to all receivers.

2. The throughput is small when the network size is increased to 300 nodes. Means MRMP multicast protocol scales well when the network size increases.

3. The average packet end-to-end delay decreases, when the number of receivers increases from 10 to 20 . The reason is that when the number of receivers increases, The SRs have more than one branches as intermediate nodes so SRs buffer the received data packets and the lost packets recovered quickly because SRs process the ACKs and retransmit repaired packets. However when the number of receivers increase continues the average EED decreases.

\section{CONCLUSION}

In this paper, we describe a cross layer reliable multicast routing protocol, the MRMP protocol. With assistance of the underlying multicast routing protocol MeshSPT, MRMP builds a recovery tree that dynamically synchronize with the multicast routing tree. Using the recovery tree the packet losses are repaired locally. through simulation results cross layer optimization in WMNs achieve high performance compare with the conventional layer.

\section{REFERENCES}

[1] Ian F. Akyildiz, Fellow, , and Xudong Wang "Cross Layer Design in Wireless Mesh Networks" IEEE TRANSACTIONS ON VEHICULAR TECHNOLOGY, VOL. 57, NO. 2, MARCH 2008.

[2] Mijahed Nasser Aljober, R. C. Thool,Atul Neg "A Sufficient and Scalable Multicast Routing Protocol in Wireless Mesh Networks MeshSPT (Shortest Path Tree algorithm for wireless Mesh network) "IJCA Proceedings on National Conference on Advancement in Electronics \& Telecommunication Engineering, 2012. 
International Journal of Distributed and Parallel Systems (IJDPS) Vol.4, No.6, November 2013

[3] Yuan, J., Li, Z., Yu, W., Li, B.: A Cross-Layer Optimization Framework for Multihop Multicast in Wireless Mesh Networks. IEEE Journal on Selected Areas in Communications 24(11) (November 2006)

[4] Rong, B., Qian, Y., Lu, K.: Enhanced QoS Multicast Routing in Wireless Mesh Networks. IEEE Transactions on Wireless Communications 7(6) (June 2008)

[5] Ehsan Pourfakhar, Amir Masoud Rahmani : Optimization of Multicast Routing Based on a Reliable effective Framework in Wireless Mesh Networks. pp. 416 - 427, 2009. Springer-Verlag Berlin Heidelberg (2009).

[6] Jorge Nuevo," A Comprehensible GloMoSim Tutorial compilation" , INRS - Universite du Quebec nuevo@inrs-telecom.uquebec.ca March 4, 2004.
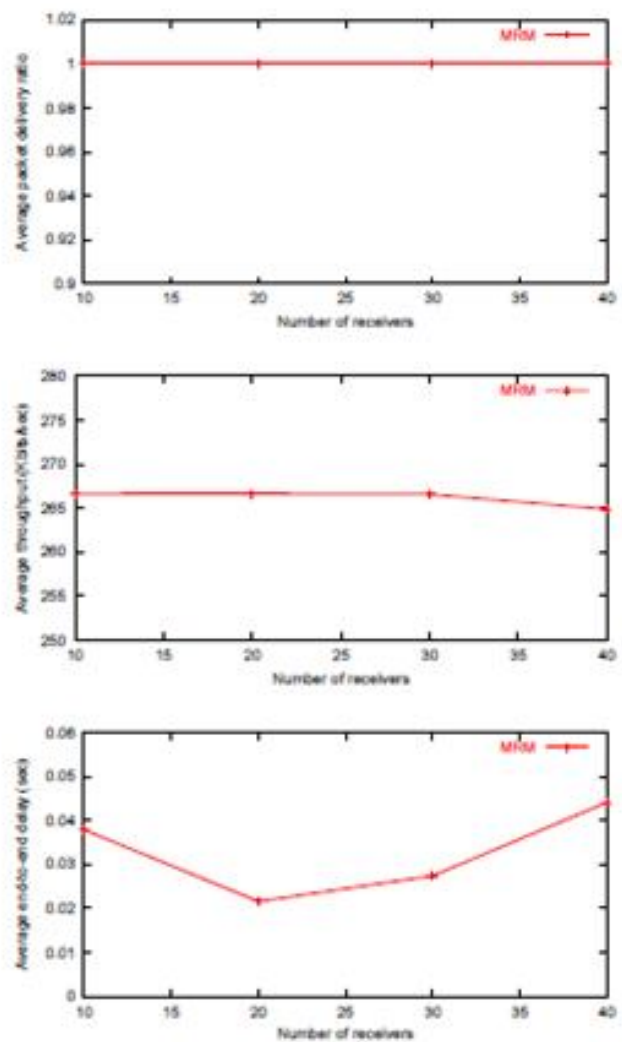

Figure 1: 100 nodes in a $2000 \mathrm{~m} * 2000 \mathrm{~m}$ terrain 
International Journal of Distributed and Parallel Systems (IJDPS) Vol.4, No.6, November 2013
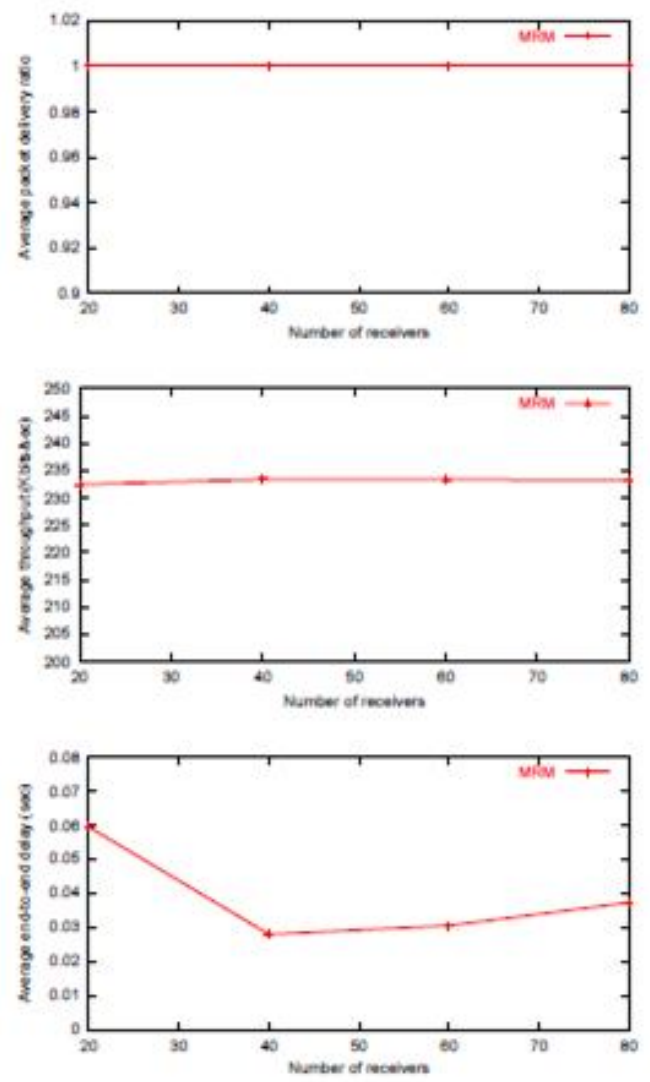

Figure 2: 300 nodes in a $3000 \mathrm{~m} * 3000 \mathrm{~m}$ terrain 